\title{
Miranda
}

Revue pluridisciplinaire du monde anglophone /

Multidisciplinary peer-reviewed journal on the English-

speaking world

\author{
16 | 2018 \\ L'expérimental dans la littérature et les arts \\ contemporains
}

\section{Compte-rendu de journée d'étude : « The Western Legacy: Hollywood Westerns and Willa Cather's My Ántonia ».}

Université de Picardie Jules Verne, Pôle Cathédrale, 16 décembre 2017/

Organisée par Marianne Kac-Vergne et Frédérique Spill

\section{Pascal Bouvet}

\section{(2) OpenEdition}

Journals

Édition électronique

URL : http://journals.openedition.org/miranda/11969

DOI : 10.4000/miranda.11969

ISSN : 2108-6559

Éditeur

Université Toulouse - Jean Jaurès

Référence électronique

Pascal Bouvet, «Compte-rendu de journée d'étude : « The Western Legacy: Hollywood Westerns and Willa Cather's My Ántonia ». », Miranda [En ligne], 16 | 2018, mis en ligne le 06 juin 2018, consulté le 16 février 2021. URL : http://journals.openedition.org/miranda/11969; DOI : https://doi.org/10.4000/ miranda. 11969

Ce document a été généré automatiquement le 16 février 2021.

\section{(†)

Miranda is licensed under a Creative Commons Attribution-NonCommercial-NoDerivatives 4.0 International License. 


\section{Compte-rendu de journée d'étude : " The Western Legacy: Hollywood Westerns and Willa Cather's My Ántonia ».}

Université de Picardie Jules Verne, Pôle Cathédrale, 16 décembre 2017/ Organisée par Marianne Kac-Vergne et Frédérique Spill

\section{Pascal Bouvet}

1 La journée d'étude « The Western Legacy: Hollywood Westerns and Willa Cather's My Ántonia » s'est déroulée le 16 décembre 2017. Elle a été organisée par Marianne KacVergne, maître de conférences en civilisation américaine et cinéma, et Frédérique Spill, maître de conférences HDR en littérature américaine, à l'Université de Picardie Jules Verne, avec le soutien du laboratoire de recherche CORPUS (Conflits, Représentations et Dialogues dans l'Univers Anglo-Saxon). Les organisatrices remercient les intervenants et les auditeurs, et plus particulièrement ceux de l'Université d'Artois avec laquelle elles souhaitent développer un partenariat dans les années à venir.

Cette journée d'étude a permis d'inviter des intervenants spécialisés dans la thématique de l'héritage de l'Ouest américain: qu'il s'agisse de cinéma ou de littérature, l'enjeu de chacune de ces interventions est celui de la présentation et de la représentation, de l'histoire et du mythe, du dit et du non-dit. Ces thématiques apportent un éclairage complémentaire pour aborder deux des questions du programme de l'agrégation externe et interne d'anglais. Le programme de civilisation américaine invite les candidats à étudier " La Construction de l'Ouest américain [1865-1895] dans le cinéma hollywoodien ». Le programme de littérature, quant à lui, inclut My Ántonia (1918) de Willa Cather, roman dans lequel le narrateur fait le récit de son arrivée au Nebraska et de sa rencontre avec Ántonia, jeune immigrée bohémienne. Le roman tisse une toile de fond faite de fiction, mais aussi de la réalité qu'a connue Cather lorsqu'elle arriva, très jeune, dans cet État des Grandes Plaines. Ainsi, qu'il 
s'agisse des films du corpus de civilisation ou du roman My Ántonia, spectateurs et lecteurs ont accès à une partie de l'héritage de l'Ouest américain.

3 La première intervention de la matinée s'intitule « Frontier Myth and Western History in the American Western Film ». Hervé Mayer, maître de conférences en civilisation américaine et cinéma à l'Université Paul Valéry Montpellier, souligne d'emblée la confusion qui règne entre histoire et mythe dans le genre qu'est le western hollywoodien. Mayer explique comment le mythe a souvent relégué l'histoire au second plan. C'est ainsi la question de l'historiographie, écriture ou réécriture de l'histoire, qui se pose. On gardera toutefois à l'esprit que l'ambition d'Hollywood n'a jamais été de produire des " cours d'histoire ", mais bien des films dont la trame narrative puise dans l'histoire de la Construction de l'Ouest américain pour divertir un public le plus large possible. Une fois cette mise en garde posée, il est possible de discerner le « mythique " (mythical genre) de ce qui relève de "l'historique " (historical genre) dans les westerns hollywoodiens. Hervé Mayer indique que la glorification de l'Ouest américain précède l'invention du cinéma et que l'on en trouve la trace dans des œuvres littéraires ou picturales (par exemple, Owen Wister et Frederic Remington) ou dans des écrits historiographiques (Turner, Roosevelt, entre autres). Tout en perpétuant cette mythification, Hollywood s'est attaché à teinter ses westerns d'une dimension historique: les studios consacrèrent une part de leur budget à la recherche sur l'histoire de l'Ouest américain, et ce afin de créer l'illusion de l'authenticité, puissant argument commercial pour promouvoir leurs productions. Les westerns dits " révisionnistes » comme Little Big Man (Arthur Penn, 1970) font la démonstration d'une prise de conscience et de distance vis-à-vis de la mythologie populaire. En ce sens, le western ne " révise » pas l'histoire à proprement parler, mais bien la manière dont elle a pu être présentée dans bon nombre des westerns au $\mathrm{XX}^{\mathrm{e}}$ siècle.

La seconde intervention est assurée par Claire Dutriaux, maître de conférences en cinéma et civilisation américaine à l'Université Paris IV-Sorbonne, et s'intitule " Law and (Dis)Order in the Westerns and the American West ». Claire Dutriaux entame sa présentation en inscrivant l'opposition " ordre vs. désordre " dans les dichotomies fondatrices du western identifiées par Jim Kitses (l'individu vs. la communauté ; l'honneur vs. les institutions ; la nature vs. la culture...). Ces dichotomies sont au cœur de la sémantique et de la syntaxe du genre (John G. Cawelti). Il n'est que de penser, par exemple, au truculent Judge Haller dans The Iron Horse (John Ford, 1924) ou encore au héros éponyme de Shane (George Stevens, 1953) : ces personnages sont des emblèmes du mythe de l'Ouest, plus que des personnages de chair. Dutriaux explique comment les westerns dépeignent une période de transition : celle consistant à structurer, ordonner, faire régner l'ordre dans l'Ouest dans l'attente de " l'arrivée de l'Est "; autrement dit, les individus qui peuplent les westerns du corpus sont souvent isolés et confrontés à une forme de violence qu'ils jugent nécessaire, et qui est ainsi mise au service de la communauté dans l'attente du développement et de la stabilisation d'institutions locales instaurées sous l'égide de l'Est, c'est-à-dire du gouvernement fédéral. Dans Broken Arrow (Delmer Daves, 1950), Jeffords est seul face à la communauté locale, mais agent facilitateur du traité de paix entre le gouvernement fédéral et les Apaches. Dans Jesse James (Henry King, 1939), la dynamique est différente : les frères James se font les défenseurs de la communauté locale contre les grandes entreprises du chemin de fer, symbole de l'ambition expansionniste du gouvernement fédéral. La violence naissant de ces tensions apparaît comme nécessaire, voire " régénérative » selon l'expression de Richard Slotkin. Les westerns contribuent à ancrer dans les esprits le mythe d'une 
justice absente, inopérante, instable. The Ox-Bow Incident (William A. Wellman, 1943) en est la parfaite illustration avec: un juge probe, mais impuissant ; un shérif absent, qui réapparaît à la fin du film; un shérif-adjoint irresponsable et expéditif. Cette représentation des figures garantes de l'autorité justifie les paradoxes sur lesquels se fonde la caractérisation des as de la gâchette (gunfighters) et des hors-la-loi : Hollywood choisit des héros complexes et ambivalents pour ses westerns. Alors que Jesse James est abattu, précisément au moment où il décide d'abandonner sa vie de banditisme, Wyatt Earp dans My Darling Clementine (John Ford, 1946) doit abandonner son rôle d'autorité de maintien de l'ordre dans la ville de Tombstone après avoir abattu les Clanton : la frontière entre l'ordre et le désordre est ainsi ténue dans les westerns et démontre que son franchissement n'exclut jamais un retour en arrière. Il s'agit d'une forme de circularité narrative propre au genre du western.

Deux intervenants prennent en charge la troisième communication: Kevin Dwyer et Julie Assouly, tous deux maîtres de conférences en civilisation américaine et cinéma à l'Université d'Artois. Cette présentation, intitulée "The Language of the Natives in Hollywood Westerns ", aborde la thématique des langues et du langage sous l'angle du verbal (Dwyer) et du non verbal (Assouly). L'étude de l'intégration de la langue dans les westerns hollywoodiens met en lumière de multiples enjeux, que résument les questions suivantes : comment s'adresse-t-on aux membres de peuples autochtones dans les westerns ? Comment s'expriment-ils ? D’emblée, Kevin Dwyer souligne la complexité des échanges interculturels et interlinguistiques lors des divers points de contact entre les peuples autochtones et les populations dites « blanches ». Le médium qu'est le cinéma a souvent opéré le choix de la simplification de ces échanges, et ce afin de satisfaire aux attentes du public. De cette simplification a pu découler la déshumanisation des Indiens dans les westerns. The Iron Horse en est une excellente illustration: la trame narrative n'accorde pas la " parole » aux Indiens, qui sont représentés comme sauvages et menaçants. Au fur et à mesure de la reconnaissance grandissante des cultures autochtones, le cinéma hollywoodien s'est attaché à placer Indiens et blancs sur un pied d'égalité : dans Broken Arrow, le pacte narratif pose dès la scène d'ouverture que les dialogues seront en anglais, y compris pour les échanges avec des Apaches. La langue anglaise permet donc aux spectateurs d'avoir un accès direct aux cultures autochtones. On note toutefois que cette stratégie narrative est à double tranchant: Jeffords adopte un ton paternaliste pour expliquer la culture " américaine " à la jeune Sonseeahray. Cette tendance s'opère au travers de la " relexification ": Sonseeahray demande la signification de termes anglais; Jeffords y accole une dénotation erronée et une connotation inaccessible à la jeune Apache, niant tout à la fois la culture de cette dernière et imposant la culture " américaine " comme seule référence. Dans la mesure où les Indiens sont privés de parole dans de nombreux westerns, l'étude du langage non-verbal que propose Julie Assouly met en lumière la caractérisation et la représentation des peuples autochtones. De nombreux procédés métonymiques visent à rendre les Indiens invisibles: par exemple, dans Stagecoach (John Ford, 1939), la musique menaçante aux sonorités indiennes ou encore la flèche qui tue à distance participent d'une réification des Indiens, lesquels ne sont que des éléments, parmi d'autres, dans la sémantique des films. Parce que la communication interlinguistique est difficilement représentable à l'écran, Hollywood recourt aux mimes, aux signaux de fumée ou de miroirs, aux cailloux pour compter les jours, aux danses rituelles sioux que des blancs sont, étonnamment, capables d'interpréter. Dwyer et Assouly notent que le western n'est pas le vecteur le plus apte à dépeindre la 
complexité d'échanges interlinguistiques, mais ils soulignent aussi la prise de conscience des réalisateurs sur cette question tout au long du XXe siècle.

6 La première intervention de l'après-midi concerne également le langage. Florent Dubois, doctorant à l'Université Paris Diderot, présente "Voice, Memory, and Language in Willa Cather's My Ántonia ». Dubois rappelle la fascination de Cather pour la voix des actrices de son enfance. De cette fascination naît chez Cather l'importance de l'oralité dans ses romans et, plus particulièrement, dans My Ántonia, dans lequel le lecteur est confronté à une œuvre très " écrite ", et pourtant prompte à laisser la place à son narrateur et au récit. Florent Dubois explique que, de manière générale, l'on se rappelle davantage le contenu sémantique d'un message que la voix qui porta ce message. Ainsi, dans My Ántonia, Jim Burden surmonte l'obstacle du temps pour non seulement rendre les paroles, le timbre des voix, mais aussi des éléments non verbaux qui participent de la symphonie des sens que crée le roman. La distinction qu'Aristote opérait entre zôe (" la vie nue ", commune à tous les êtres vivants) et bios (" la vie sociale ", propre aux êtres humains) permet à Dubois de déceler dans My Ántonia diverses sources de vie et, donc, de voix. On attribuait volontiers le concept zồ aux animaux, car on les jugeait pourvus d'une " voix ». Dès lors, la polyphonie s'élargit audelà des personnages : les abeilles lors du pique-nique au bord la rivière (" I could hear the bees humming and singing », p. 129), les coyotes alors que Pavel est mourant ("The coyotes broke out again; yap, yap, yap-then the high whine. ", p. 35) ou, encore, les loups et les chevaux durant l'épisode du traîneau ("The screams of the horses were more terrible to hear than the cries of the men and women. ", p. 37). Cather transcende ce concept et, par un jeu de métaphores, donne vie et voix à l'inanimé : les outils d'Otto Fuchs sur les planches du cercueil de Mr. Shimerda (« one could hear the panting wheeze of the saw or the pleasant purring of the plane. », p. 63). Les sons ont ainsi le pouvoir magique d'évoquer le passé - en particulier, lorsque les mots viennent à manquer. La stridulation d'une sauterelle, par exemple, rappelle à Ántonia un personnage de son enfance (Old Hatta, p. 27) et la rend mélancolique. Dubois voit dans cette polyphonie l'ambition esthétique de Willa Cather (dont elle jette les fondements dans son essai "The Novel Démeublé ", 1922), qui n'appréciait que peu les descriptions du roman moderne. Cather propose un roman dont le projet est d'être une expérience des sens, un roman « évocateur » de l'enfance du narrateur et de sa propre enfance dans l'Ouest américain.

7 Françoise Palleau, professeur en littérature américaine à l'Université Paris 13, spécialiste de l'œuvre de Willa Cather, conclut cette journée d'étude avec une présentation intitulée "When text becomes metatext in My Ántonia ». Palleau pose l'axe d'analyse suivant: dans quelle mesure certains passages du roman reflètent-ils les intentions de Cather ? La métatexualité dans My Ántonia s'inscrit dans un jeu d'écriture permettant à Cather de rédiger un roman qui relate des épisodes faisant écho à des éléments et des personnages structurants de la trame narrative : le Livre $\mathrm{V}$ est émaillé de références aux quatre précédents. Il est à nouveau question de la voix quand il s'agit ici d'analyser la métatextualité dans My Ántonia. Lorsque Jim se souvient avec émotion de la voix de son grand-père, lequel lisait des passages de la Bible (" His voice was so sympathetic and he read so interestingly that I wished he had chosen one of my favorite chapters in the Book of Kings. ", p. 14), Palleau démontre comment cette incarnation de la sagesse, de la piété et de la compassion, devient elle-même la représentation de Dieu dans le roman. La caractérisation de ce personnage, et des autres, témoigne ainsi du travail d'écriture et de la dimension qui échappe au " dit »: c'est-à-dire, la métatextualité. Willa Cather parvient ainsi à transmettre des émotions à ses lecteurs sans verser dans 
le sentimentalisme. La patria des Géorgiques de Virgile est à la fois l'inspiration de Gaston Cleric et la source des émotions intenses qu'il ressent. La patria réside au cœur du récit porté par le narrateur qui s'interroge à de nombreuses reprises sur sa place : « Out there I felt at home again. ", p. 195. Françoise Palleau établit le lien entre patria et le mot anglais home. Place du narrateur, mais aussi place du lecteur: la dimension métatextuelle de l'écriture de My Ántonia permet au narrateur, par le choix de ses mots, de dialoguer directement avec son lecteur implicite. L'emploi récurrent du pronom indéfini one, par exemple, est source de connivence avec le lecteur : " that is happiness; to be dissolved into something complete and great. When it comes to one, it comes as naturally as sleep. ", p. 17. Par là même, tout en conservant leur caractère personnel, les souvenirs de Jim acquièrent une dimension transpersonnelle. Qu'il s'agisse du vagabond qui se suicide sous les yeux d'Ántonia ou, encore, des personnages pathétiques de la pièce Camille $^{1}$ à laquelle assistent Lena et Jim, ces épisodes sont avant tout une mise en perspective métatextuelle de la vie des personnages du roman. Cette dernière présentation de la journée se conclut par un échange visant à affiner plus encore la réflexion des auditeurs sur l'écriture de Cather : Palleau et Dubois soulignent la volonté et l'ambition de Cather d'écrire des romans s'adressant à un lectorat le plus large possible, et ce malgré ses propres goûts, dont ils s'accordent à dire qu'ils étaient élitistes. De la même manière, Cather crée un narrateur capable de mettre entre parenthèses sa sensibilité pour faire le récit de son Ántonia et l'inscrire dans une expérience sensuelle de l'Ouest américain.

\section{BIBLIOGRAPHIE}

Cather, Willa. My Ántonia [1918]. Janet Sharistanian, ed. Oxford : Oxford University Press (Oxford World's Classics), 2006.

\section{NOTES}

1. Titre anglais de l'adaptation théâtrale du roman La Dame aux camélias d'Alexandre Dumas fils. 
INDEX

Mots-clés : Frontière, mythe, histoire, historiographie, western hollywoodien, ordre et désordre, peuples autochtones, langues, langage verbal, langage non-verbal, Willa Cather, My Ántonia, voix, mémoire, métatextualité, Little Big Man, The Ox-Bow Incident, Jesse James, Broken Arrow, My Darling Clementine, The Iron Horse, Stagecoach, Shane, Géorgiques

Keywords : Frontier, myth, history, historiography, Hollywood Western, law and (dis)order, native peoples, languages, verbal language, non-verbal language, Willa Cather, My Ántonia, voice, memory, metatextuality, Little Big Man, The Ox-Bow Incident, Jesse James, Broken Arrow, My Darling Clementine, The Iron Horse, Stagecoach, Shane, Georgics

Thèmes : Film, TV, Video

\section{AUTEURS}

\section{PASCAL BOUVET}

Professeur agrégé

Université de Picardie Jules Verne

pascal.bouvet@ac-amiens.fr 